\section{Entwicklung klinischer Studien von Paul Martini bis heute}

\author{
M. Schumacher ${ }^{1}$ \\ ${ }^{1}$ Institut für Medizinische Biometrie und Statistik, Medizinische \\ Fakultät der Universität Freiburg und Universitätsklinikum \\ Freiburg
}

Als ich Anfang 1979 an das Institut für Medizinische Dokumentation, Statistik und Datenverarbeitung der Universität Heidelberg unter der Leitung von Professor Herbert Immich, einem der Wegbereiter der Medizinischen Statistik in Deutschland [1], kam, herrschte dort eine gewisse „Aufbruchsstimmung“ hinsichtlich der Planung und Durchführung größerer klinischer Studien. Im Jahr zuvor war unter Federführung von Professor Hans Joachim Jesdinsky das „Memorandum zur Planung und Durchführung kontrollierter klinischer Therapiestudien“ [2] erschienen; gleichzeitig hatte im Rahmen des „Programms der Bundesregierung zur Förderung von Forschung und Entwicklung im Dienste der Gesundheit“ [3] eine Ausschreibung zum Aufbau von sogenannten Methodischen Zentren und zur Förderung von größeren Multicenterstudien stattgefunden. Immich hatte an dem Memorandum mitgewirkt, und das Heidelberger Institut hatte den Zuschlag für ein Zentrum zur methodischen Betreuung von Therapiestudien beim Mammakarzinom bekommen. Der Senior der Arbeitsgruppe, Dr. Hans Scheurlen, der als Radiologe selbst die „Heidelberger Studie“ [4] mit 142 Patientinnen durchgeführt hatte, war sich deren methodischer Unzulänglichkeiten durchaus bewusst und sorgte dafür, dass ich mich vom ersten Tag an mit dieser Thematik beschäftigte. Diese Beschäftigung hat dann viele Jahre angedauert, und die Methodik klinischer Studien hat einen großen Teil meiner akademischen Laufbahn bestimmt [5]. Die Studien der „German Breast Cancer Study Group“ wurden nach langwieriger Vorbereitungsphase tatsächlich erfolgreich durchgeführt und mit Publikation der Langzeitergebnisse viele Jahre später abgeschlossen [6].

Doch warum war zu dieser Zeit ein großangelegtes Förderprogramm [3] notwendig und warum war das Memorandum [2] so wichtig? Dazu müssen wir in die 1930er Jahre zu Paul Martini und Sir Austin Bradford Hill zurückgehen. 1932 hatte Paul Martini, gerade auf den Lehrstuhl der Medizinischen Klinik in Bonn berufen, die „Methodenlehre der therapeutischen Untersuchung “ [7] veröffentlicht. Ausgehend von einer Kritik an den von ihm festgestellten damaligen Missständen hat er dort „Kriterien für die Durchführung klinisch-therapeutischer Studien“ entworfen [8]. Das „1. Grundgesetz“ ist dabei der therapeutische Vergleich, der kollektiv oder auch individuell durchgeführt werden kann (in heutiger Terminologie wäre das ein Parallel-Gruppen-Design bzw. eine CrossOver-Studie). Das „2. Grundgesetz“ ist die Ausschaltung von Mitursachen, z.B. durch sogenannte unwissentliche Versuchsanordnungen wie etwa durch Verblindung [9]. Was für ein heutiges Studiendesign fehlt, ist die Randomisation, d.h. die zufällige Zuteilung der Patienten zu den Behandlungsmodalitäten; stattdessen schlug Martini die „Anwendung einer ausgleichenden Alternierung“ [9] vor. Bei Bradford Hill [10,11] im Jahr 1937 ist die Randomisation zur Vermeidung eines Selektions-Bias dagegen integraler Bestandteil der Methodik für therapeutische Vergleiche, die ansonsten sehr ähnliche Kriterien wie die von Martini angeführten umfasst. Bradford Hill konnte seine Ideen und Vorstellungen dann im Jahre 1948 umsetzen in einer größeren klinischen Studie zur Behandlung der pulmonalen Tuberkulose [12], die gemeinhin als die erste moderne kontrollierte klinische Studie angesehen wird [13], die auch heutigen Qualitätsanforderungen weitestgehend standhalten würde.

Springen wir ins Jahr 1966, das Gründungsjahr der Paul-Martini-Stiftung, zwei Jahre nach seinem Tod. Ein nicht-systematischer Querschnitt durch die damalige, meist deutschsprachige klinische Literatur zeigt einige Arbeiten zur Methodik klinischer Studien, insb. zur Problematik des DoppelblindVersuchs, sowie einige Berichte zu kleineren, von den Autoren selbst durchgeführten klinischen Studien [14-18]. Bemerkenswert dabei ist, dass die Randomisation nicht explizit angeführt wird, ein „zufälliges Element“ eher indirekt bei der Durchführung der Verblindung, z. B. bei der Vorbereitung der Studienmedikation eingebracht wird [14]. Dies ist umso erstaunlicher, weil es in der Zwischenzeit im angelsächsischen Sprachraum eine ganze Reihe prominenter randomisierter kontrollierter Studien gab, z.B. die bekannte Salk-Polio-Studie mit mehreren hunderttausend teilnehmenden Kindern $[5,19]$. In einer der letzten Publikationen von Paul Martini, der schriftlichen Fassung seines Einführungsvortrags zu einem Seminar über klinische Studien [9], gibt es einen Hinweis „...Ins Praktische übersetzt, heißt es, dass bei der Versuchsplanung der kollektiven Vergleiche nur der Zufall darüber entscheiden darf, wie jede der beiden zu vergleichenden Gruppen zustande kommt“, der jedoch im nachfolgenden Satz „Dieser Forderung des Zufalls widerspricht nicht die Modifikation der ausgleichenden Alternierung;... “ zumindest etwas abgeschwächt wird.

Ein Schüler von Paul Martini war es dann, der maßgeblich zu einer Zusammenführung dieser beiden „Richtungen“ beigetragen hat. Hans Joachim Jesdinsky hatte in den 1950er Jahren bei Paul Martini promoviert und war dann nach kurzer Tätigkeit in der pharmazeutischen Industrie am Institut für Medizinische Statistik und Dokumentation der Universität Freiburg unter Professor Edward Walter [20] tätig. Hier habilitierte er sich 1969 mit einer Arbeit zur Versuchsplanung in der Medizin [21,22] und unterstützte aktiv die Planung, Durchführung und statistische Auswertung von randomisierten Therapiestudien. Ein Beleg dafür ist die im New England Journal of Medicine publizierte Studie einer europäischen Studiengruppe zur Streptokinase-Behandlung des akuten Myokardinfarkts [23], bei der Jesdinsky zusammen mit weiteren Freiburger Kollegen für die statistische Planung und Auswertung zuständig war. Nach der Übernahme des Lehrstuhls für Medizinische Statistik in Düsseldorf im Jahr 1975 hat er dann federführend eine Arbeitsgruppe, der fast alle damaligen Fachvertreter und Vertreter der pharmazeutischen Industrie angehörten, geleitet, die das bereits zu Beginn erwähnte Memorandum [2] erstellt und 1978 herausgegeben hat. Dieses läutete dann das Zeitalter größerer randomisierter klinischer Multicenter-Studien in Deutschland 
ein. Jesdinskys früher Tod im Jahr 1986 hat dann seinen Anstrengungen, der klinischen Forschung weitere Impulse zu geben, ein jähes Ende gesetzt [21,22].

Der Prototyp der randomisierten klinischen Studie hat als „Gold-Standard“ in den letzten 40 Jahren das Bild der klinischen Therapieforschung weltweit und auch in Deutschland bestimmt. Dabei können, soweit möglich, zusätzliche Verblindungs- und Maskierungstechniken zum Einsatz kommen. Im Bereich der Arzneimittelstudien gibt es zahlreiche gesetzliche Vorgaben und im Rahmen der Zulassung wichtige Richtlinien zu beachten, die ständig hinsichtlich neuer Anforderungen, wie etwa der Betrachtung von komplexen Ereigniszeitendpunkten, aktualisiert und weiterentwickelt werden. Die Auswahl der Studiendesigns beschränkt sich dabei im Wesentlichen auf einige wenige: das 2-Gruppen-Design mit Vergleich zur Standardtherapie (manchmal auch auf mehr als 2 Gruppen erweitert), das „Dose-Response“-Design mit mehreren Dosierungsstufen, das faktorielle Design zur Untersuchung der verschiedenen Kombinationen von Therapiemodalitäten sowie das Cross-Over-Design mit intraindividuellem Vergleich in Situationen, wo dies angezeigt ist (z.B. bei nur langsam fortschreitender chronischer Erkrankung und symptomatischer Behandlung). Alle diese „klassischen“ Ansätze sind auch heute noch aktuell und werden in großem Umfang eingesetzt, was sich in der ständig wachsenden Anzahl von registrierten Studien (z.B. in clinicaltrials.gov oder dem Deutschen Register Klinischer Studien) widerspiegelt.

Man könnte also meinen, die Methodik klinischer Studien befände sich in einer Art „Gleichgewichtszustand“, an dem es nur noch kleinere Dinge zu verändern bzw. zu verbessern gilt. Dies ist jedoch mitnichten so: mit der Ankunft der Präzisionsmedizin (oft auch als individualisierte oder personalisierte Medizin bezeichnet), die sich zum Ziel gesetzt hat, den individuellen Patienten mit Hilfe von genetischer oder anderer molekularer Information mit dem richtigen Medikament, der richtigen Dosis und zum richtigen Zeitpunkt optimal zu behandeln, haben sich große Herausforderungen ergeben, denen man nicht nur mit marginalen Änderungen Rechnung tragen kann. Ich möchte das am Beispiel der vor etwa einem Jahr publizierten SHIVA-Studie [24] zur molekularen, zielgerichteten Therapie für Patienten mit fortgeschrittener Tumorerkrankung veranschaulichen. Bei diesem sogenannten „alltumor trial“ werden Patienten mit soliden und bereits therapie-refraktären Tumoren im metastasierenden Stadium aufgenommen. Nach der Sequenzierung des durch Biopsie gewonnenen Tumormaterials und entsprechender bioinformatischer Aufbereitung wird in einem Molekularen TumorBoard überprüft, ob für die gefundenen molekularen Veränderungen eine zielgerichtete Therapie zur Verfügung steht oder nicht. Falls ja, wird der Patient nach erfolgter Aufklärung randomisiert zu entweder der passenden molekularen, zielgerichteten Therapie oder der nach Einschätzung des behandelnden Onkologen bestmöglichen konventionellen Therapie. Insgesamt wurden knapp 200 Patienten in dieser Phase 2Studie randomisiert, die ein breites Spektrum an Tumorentitäten und an molekularen Veränderungen aufwiesen [25]. Die Ergebnisse dieser Studie waren enttäuschend, zeigte sich doch kein Unterschied hinsichtlich der progressionsfreien Überlebenswahrscheinlichkeit zwischen den beiden Thera- piegruppen. Eine Analyse in drei nach molekularen Kriterien gebildeten Subgruppen ergab einen allerdings statistisch nicht-signifikanten Hinweis auf eine mögliche Überlegenheit der zielgerichteten Therapie in einer der drei (und zwar der kleinsten) gebildeten Subgruppen. Aus dem Design und der statistischen Auswertung der SHIVA-Studie konnten eine Reihe von Problemfeldern identifiziert werden, die derzeit diskutiert werden und hoffentlich zur verbesserten Konzipierung von zukünftigen Studien im Bereich der Präzisionsmedizin führen werden [24].

Zusätzlich zu den gerade geschilderten Themen kommen viele weitere im Bereich von Studien in der Präzisionsmedizin hinzu, die hier aufzuzählen den Rahmen dieses Beitrags sprengen würde $[26,27]$. Es ist aber wichtig, festzustellen, dass die Grundprinzipien der Methodik klinischer Studien, so wie sie von Martini und Bradford Hill formuliert und später vom Martini-Schüler Jesdinsky als Voraussetzung für die breite Anwendung in Deutschland zusammengeführt wurden, weiterhin beachtet werden müssen, auch wenn neue Elemente bei Design und Auswertung hinzukommen [27]. Ich möchte deshalb auf den letzten Absatz in der bereits erwähnten Arbeit von Martini [9] verweisen:

„Seit Urzeiten wurde ganz unverdientermaßen völlig kritiklosen Urteilen über Heilmittel der Ehrenname, Erfahrung zugestanden. Diese Erfahrung war aber keine echte, sondern nur eine naive und deshalb ganz und gar unzuverlässige Erfahrung. Sie war die Gefahr, die die therapeutische Forschung in der hinter uns liegenden Zeit bedrohte. Für die zukünftige therapeutische Forschung aber sehe ich auch eine Gefahr. Ich sehe sie in dem Abgleiten in eine Selbstgenügsamkeit, die schon in der Erfüllung der formalen Bedingungen und in formaler Exaktheit ihre Befriedigung findet, die aber die äußerste und redlichste Genauigkeit in Bezug auf die individuelle Analyse des einzelnen Kranken weniger hoch in Anschlag bringen könnte.“

In diesem Sinne sollte es uns gemeinsam gelingen, den neuen Anforderungen, die sich aus den Zielen der Präzisionsmedizin ergeben, durch Weiterentwicklung von adäquater Methodik bei Design, Durchführung und Auswertung von klinischen Studien Rechnung zu tragen und damit deren Ergebnisse auf ein „evidenzbasiertes Fundament“ zu stellen.

\section{Interessenkonflikt: Nein}

\section{Literatur}

1 Schumacher M. Methodische Grundlagen klinischer Forschung - Reminiszenzen an Herbert Immich und seine Beiträge zur Medizinischen Statistik. Informatik, Biometrie und Epidemiologie in Medizin und Biologie 2004; 35: 44-53

2 Jesdinski HJ, Hrsg. Memorandum zur Planung und Durchführung kontrollierter klinischer Therapiestudien. Schriftenreihe der GMDS, Heft 1. Stuttgart, New York: Schattauer; 1978

3 Der Bundesminister für Forschung und Technologie, Hrsg. Programm der Bundesregierung zur Förderung von Forschung und Entwicklung im Dienste der Gesundheit 1978-1981. Bonn; 1978

4 Friedl W, Scheurlen HR, Henningsen B. Der Wert der Strahlentherapie nach modifiziert radikaler Mastektomie. 10-Jahres-Ergebnisse der Heidelberger Studie. DMW 1983; 108: 325-330

5 Schumacher M, Schulgen G. Methodik klinischer Studien. Methodische Grundlagen der Planung, Durchführung und Auswertung. 3. Aufl. New York, Heidelberg: Springer; 2008

6 Schmoor C, Olschewski M, Sauerbrei Wet al. Long-term follow-up of patients in four prospective studies of the German Breast Cancer Study Group (GBSG): A summary of key results. Onkologie 2002; 25: $143-150$ 
7 Martini P. Methodenlehre der Therapeutischen Untersuchung. Berlin, Heidelberg: Springer; 1932

8 Stoll S. Klinische Forschung und Ethik bei Paul Martini. ZaeFQ 2003; 97: 675-679

9 Martini P. Grundsätzliches zur therapeutisch-klinischen Versuchsplanung. Methods Inf Med 1962; 1: $1-5$

10 Hill AB. Principles of medical statistics. XII - Common fallacies and difficulties. Lancet 1937; 1: 706-708

11 Hill $A B$. Controlled Clinical Trials. Oxford: Blackwell; 1960

12 Hill AB. The clinical trial. Br Med Bull 1951; 7: 278-282

13 BMJ. The randomised controlled trial at 50. BMJ 1998; 317: $1167-$ 1248

14 Weyer H, Muth HW. Das protrahiert wirkende Schlafmittel Revonal im doppelten Blindversuch. MMW 1966; 4: 217-219

15 Staemmler H-J, Schneider HPG, Sachs L. Die medikamentöse Anregung der Ovulation im doppelten Blindversuch. Klin Wschr 1966; 44: $818-824$

16 Zürcher $H-P$. Klinische Testung im Doppelblindversuch mit Sinecod ${ }^{\circledR}$ Hommel. Praxis 1966; 48: 1402 - 1408

17 Von Eiff AW, Jesdinsky HJ, Hennekeuser $H-H$ et al. Doppelter oder einfacher Blindversuch im kurzdauernden pharmakologischen Experiment? Der Effekt von Psychopharmaka auf die vegetativen Funktionen gesunder Versuchspersonen. Klin Wschr 1966; 44: 1224-1229

18 Harder A. Probleme der Prüfung von Antidepressiva am Beispiel eines neuen Dibenzodiazepinderivats. Schweiz Med Wschr 1966; 6: 185 190

19 Meier P. The biggest public health experiment ever: the 1954 field trial of the Salk Poliomyelitis vaccine. In: Tanur JM, Mosteller F, Kruskal WH, Lehmann EL, Link RF, Pieters RS, Rising GR, eds. Statistics: A Guide to the Unknown. Monterey: Wadsworth \& Brooks; 1989

20 Schumacher M. Wie die Medizinische Statistik nach Freiburg kam. Eine historische Perspektive. GMS Medizinische Informatik, Biometrie und Epidemiologie 2005; 1: DOC-07

21 Wester S, Plassmann M. Univ.Prof. Dr. Hans-Joachim Jesdinsky und die Einführung der medizinischen Statistik an der Universität Düsseldorf. In: Jahrbuch der Heinrich-Heine-Universität Düsseldorf 2007/2008. Düsseldorf: düsseldorf university press; 2008: 727-742

22 Trampisch HJ, Hrsg. Gedenkschrift anlässlich des Kolloquiums für Herrn Prof. Dr. med. Hans-Joachim Jesdinsky ( ${ }^{*} 27$. Januar 1931 - †30. Oktober 1986). Düsseldorf; 1987
23 European Cooperative Study Group for Streptokinase Treatment in Acute Myocardial Infarction. Streptokinase in acute myocardial infarction. N Engl J Med 1979; 301: 797-802

24 Le Tourneau C, Delord J-P, Gonçalves A et al. Molecularly targeted therapy based on tumour molecular profiling versus conventional therapy for advanced cancer (SHIVA): a multicentre, open-label, proof-of-concept, randomised, controlled phase 2 trial. Lancet Oncol 2015; 16: $1324-1334$

25 Le Tourneau C, Kamal M, Trédan $O$ et al. Designs and challenges for personalized medicine studies in oncology: focus on the SHIVA trial. Targ Oncol 2012; 7: 253-265

26 Matsui S, Buyse M, Simon R et al. Design and Analysis of Clinical Trials for Predictive Medicine. Boca Raton, FL: Chapman and Hall/CRC; 2015

27 Deutsche Akademie der Technikwissenschaften, Union der deutschen Akademien der Wissenschaften, Hrsg. Individualisierte Medizin. Voraussetzungen und Konsequenzen. Halle (Saale): Nationale Akademie der Wissenschaften Leopoldina, acatech; 2014

Bibliografie

DOI http://dx.doi.org/10.1055/s-0042-112398

Drug Res 2016; 66, Suppl. 1: S5-S7

(c) Georg Thieme Verlag KG Stuttgart · New York .

ISSN 2194-9379

Korrespondenzadresse

Prof. Dr. Martin Schumacher

Direktor des Instituts für Medizinische

Biometrie und Medizinische Informatik

Universitätsklinikum Freiburg

Stefan-Meier-Str. 26

79104 Freiburg

ms@imbi.uni-freiburg.de

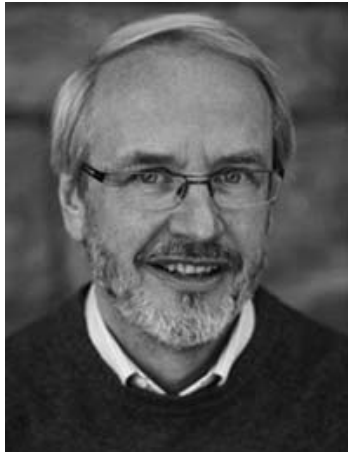

